\title{
Primary calibration of mechanical sensors with digital output for dynamic applications
}

\author{
Benedikt Seeger ${ }^{1}$, Thomas Bruns ${ }^{1}$ \\ ${ }_{1}$ Physikalisch-Technische Bundesanstalt, Bundesallee 100, 38116 Braunschweig, Germany
}

\section{ABSTRACT}

This article tackles the challenge of the dynamic calibration of modern sensors with integrated data sampling and purely digital output for the measurement of mechanical quantities like acceleration, angular velocity, force, pressure, or torque. Based on the established calibration methods using sine excitation, it describes an extension of the established methods and devices that yields primary calibration results for the magnitude and phase of the complex transfer function. The system is demonstrated with a focus on primary accelerometer calibrations but can easily be transferred to the other mechanical quantities. Furthermore, it is shown that the method can be used to investigate the quality and characteristics of the timing for the internal sampling of such digital output sensors. Thus, it is able to gain crucial information for any subsequent phase-related measurements with such sensors.

Section: RESEARCH PAPER

Keywords: Primary calibration; MEMS; complex frequency response; digital interface; dynamic measurement

Citation: Benedikt Seeger, Thomas Bruns, Primary calibration of mechanical sensors with digital output for dynamic applications, Acta IMEKO, vol. 10, no. 3, article 24, September 2021, identifier: IMEKO-ACTA-10 (2021)-03-24

Section Editor: Francesco Lamonaca, University of Calabria, Italy

Received February 26, 2021; In final form July 9, 2021; Published September 2021

Copyright: This is an open-access article distributed under the terms of the Creative Commons Attribution 3.0 License, which permits unrestricted use, distribution, and reproduction in any medium, provided the original author and source are credited.

Funding: This project 17IND12 Met4FoF has received funding from the EMPIR programme co-financed by the Participating States and from the European Union's Horizon 2020 research and innovation programme.

Corresponding author: Thomas Bruns, e-mail: thomas.bruns@ptb.de

\section{INTRODUCTION}

More and more applications and appliances in both the industrial and the consumer fields are integrating micromechanical sensors with a direct digital output. That means the measured value of a quantity like acceleration, air pressure or force is accessible via a digital interface like SPI, I2C or CAN in terms of a binary number. Those values can be directly fed into control units or digital displays for further processing without any need for traditional signal conditioning by amplifiers or sampling by discrete AD converters.

This is very beneficial for the integration of measurement capabilities in all kinds of devices and systems, from simple telephones to UAVs ${ }^{1}$ and beyond. Accordingly, the market for MEMS sensors has been booming for several years.

In condition monitoring applications, e.g., on production machines, these sensors can be used to measure vibrations, also using information from the frequency domain [1], which makes

\footnotetext{
${ }^{1}$ Unmanned aerial vehicles also known as drones.
}

dynamic calibration and absolute timestamping advantageous [2], [3].

From the metrology perspective, such sensors are rather inconspicuous as long as the focus is on a static measurement. As soon as the focus shifts onto dynamic signals, however, the timing of signal acquisition is of high importance, and the integrated sampling and signal conditioning of such sensors creates new challenges for the calibration lab.

This starts with the fact that existing documentary standards and guidelines for dynamic calibration, like the current ISO 16063 series or [4] rely on the synchronised timing of the (analogue input) data acquisition channels, which is under the control of the calibration system. Recent work on the calibration of sensors for other mechanical quantities like force, torque or pressure relies on the same principles.

While the calibration of the magnitude of a sensor's complex transfer function only needs the technical interfaces and a stationary excitation signal [5], the phase or group delay calibration is strongly dependent on either the synchronicity of the channels or other precise knowledge of the sample timing. 


\section{EXTENSION TO CONVENTIONAL PRIMARY CALIBRATION}

A conventional analogue calibration system (ACS) in compliance with ISO $16063-11$ or ISO $16063-21$ is based on two data acquisition channels with common timing usually provided by a central clock signal in a single data acquisition system. The two channels record the reference quantity $\left(y_{\text {ref }}\right)$, like acceleration, velocity, or displacement) and the calibration quantity (voltage, charge) as the output of the device under test (DUT).

The clock is usually quite accurate and the sampling of the time series from the acquisition can be considered equidistant.

Using a mono-frequent sinusoidal drive signal with angular frequency $\omega$ for the mechanical excitation (e.g., via a shaker), a linear sine approximation is used to quantify the reference input $\left(y_{\text {ref }}\right)$ and the response $\left(y_{\text {DUT }}\right)$ of the DUT. Via linear least squares fitting, the parameters in the following equations are determined:

$$
\begin{aligned}
& y_{\text {ref }}\left(t_{i}\right)=A_{\text {ref }} \sin \left(\omega t_{i}\right)+B_{\text {ref }} \cos \left(\omega t_{i}\right)+C_{\text {ref }} \\
& y_{\text {DUT }}\left(t_{i}\right)=A_{\text {DUT }} \sin \left(\omega t_{i}\right)+B_{\text {DUT }} \cos \left(\omega t_{i}\right)+C_{\text {DUT }},
\end{aligned}
$$

where $A$ and $B$ can be considered as quadrature components of each signal, while $C$ is introduced to cover any potential bias introduced into the measurement.

The complex transfer function $S$ of the DUT is then given in terms of magnitude $\hat{S}$ and phase $\Delta \varphi$ by

$$
\begin{aligned}
& S(\omega)=\hat{S}(\omega) \cdot \mathrm{e}^{-i \Delta \varphi(\omega)} \text { with } \\
& \hat{S}(\omega)=\sqrt{\frac{A_{\mathrm{DUT}}^{2}+B_{\mathrm{DUT}}^{2}}{A_{\mathrm{ref}}^{2}+B_{\mathrm{ref}}^{2}}} \text { and } \\
& \varphi(\omega)=\arctan \left(\frac{B_{\mathrm{DUT}}}{A_{\mathrm{DUT}}}\right)-\arctan \left(\frac{B_{\mathrm{ref}}}{A_{\mathrm{ref}}}\right) .
\end{aligned}
$$

Although not self-evident from the equations, it is crucial for the correct calibration of the phase delay that the time base for the data acquisition (represented by $t_{i}$ in equation (1) is the same for both channels.

In Figure 1, such an ACS is represented on the top left. The channel for the DUT called "sync" in this schematic for reasons that will become clear, soon.

\subsection{Sample timestamping}

For the case of a digital output sensor (DOS), the prerequisite last mentioned in the previous paragraph is no longer fulfilled. Neither is the sample clock frequency (or the actual instance of sampling) under the control of the laboratory's ACS nor is the exact instance of sampling even known to the measurement system.

A reliable phase calibration under such circumstances requires an extension of the ACS in order to mitigate the lack of knowledge and control.

For the rest of this section it is presumed that the DOS provides a "data ready signal", that is, it provides a means to signal to any connected recording system that a new sample has been acquired and is ready for delivery. This signal is active whenever a new sample was acquired by the sensor.

The term "sample" in relation to the DOS relates to one set of values of the measurands of the DOS. These values are nominally associated with the same single point in time, e.g., for a three-component accelerometer, a sample could be a tuple $\left(a_{x}, a_{y}, a_{z}\right)$.

The complications compared to a conventional analogue sensor are threefold:

1. The data interface is digital and cannot be read out by the classic ACS.

2. The precise sample clock is unknown and asynchronous in relation to the ACS's internal clock.

3. The sampling instances of the DOS are not necessarily equidistant.

To overcome these complications and accomplish a phase calibration of a DOS with data ready output, the ACS is extended with a separate digital acquisition unit (DAU) as shown in the top right part of Figure 1.
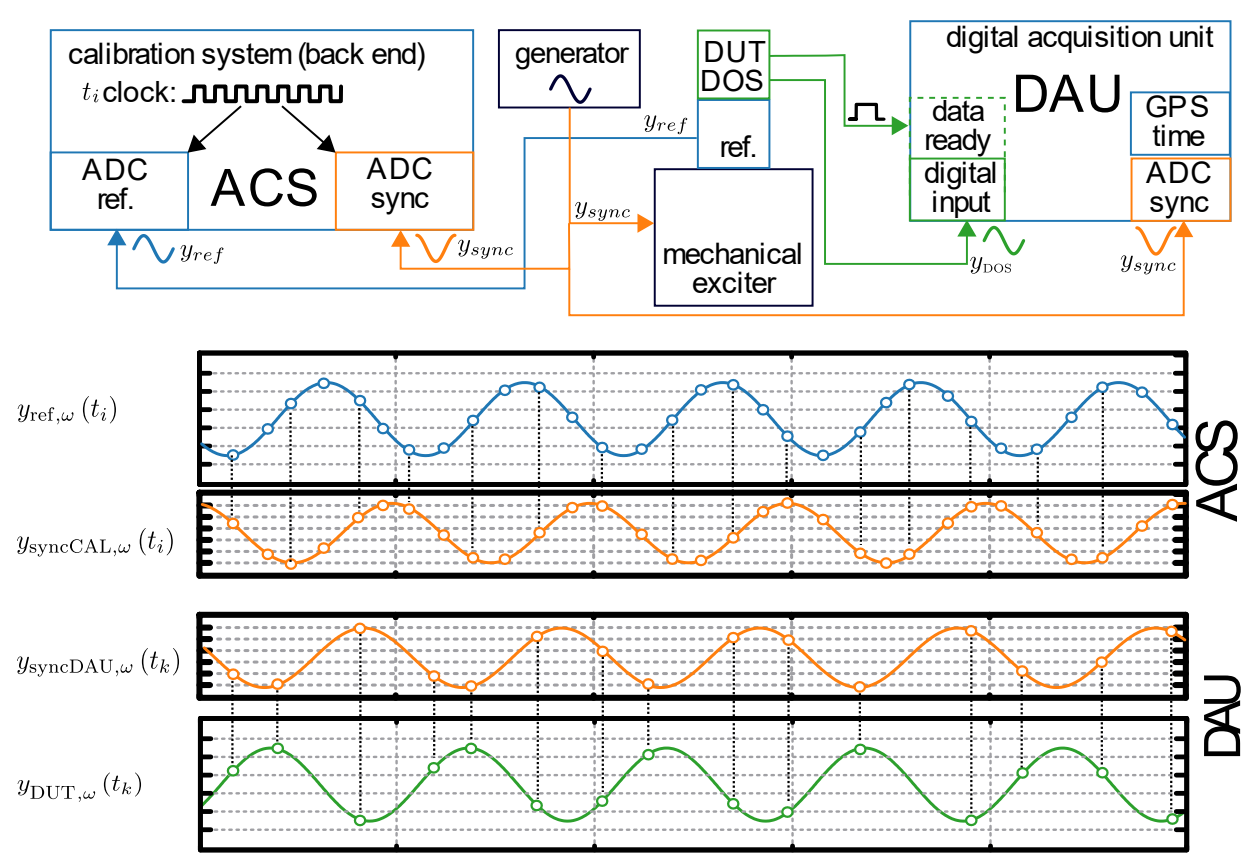

Figure 1. The conventional analogue calibration system (ACS) on the top left extended by the digital acquisition unit (DAU) on the top right. At the bottom the respective signal traces over time as acquired by the two systems. 
The core of this DAU is the sampling and timestamping of the data from the digital interface of the DOS related to a common time base, which it also uses for the sampling of an additional analogue channel called "sync" (orange arrows) in Figure 1.

In the implementation used for the investigations in this publication, the sampling of DOS data and sync were performed synchronously. This, however, is not a strict requirement for the procedure.

Whenever the DOS signals the availability of a new sample via the data ready output (upper green arrow in Figure 1, the DAU immediately samples the sync signal, $\left(y_{\text {sync }}\right)$ (right orange input to DAU in Figure 1), reads the digital data sample, ( $\left.y_{\mathrm{DOS}}\right)$ from the DOS interface (lower green arrow in Figure 1) and marks this data tuple with a precise timestamp based on a GPS aligned clock of the microprocessor used.

Repeating this procedure, the DAU acquires a time series of digital and analogue data with a sample clock as close to the DOS internal sample clock as possible but with timing information as accurate as the external clock provided to the DAU.

The relation to the reference signal for the calibration, which is still acquired with the ACS (blue arrow in Figure 1), is provided by a sync signal (orange arrow) and ACS input in Figure 1, hence the naming of this channel.

The sync signal is a mono-frequent sinusoidal voltage with the identical frequency to the excitation signal. It is fed into the DUT channel of the ACS and to the sync input of the DAU.

Such a signal can be easily derived directly from the excitation signal or taken from the reference channel of the ACS.

As a consequence of this setup and procedure, the originally independent systems of the ACS and the DAU can now be linked in time via the common sync signal which is sampled by both systems based on their respective time bases. This is depicted in the time traces in the lower part of Figure 1, where the same sync signal (in orange) is given twice: once as acquired by the ACS and secondly as acquired by the DAU.

\subsection{Implementation details of the DAU}

The implementation of the DAU used in this study was based on an STM $32 \mathrm{~F} 767^{\circledR}$ microcontroller and a related development board (NUCLEO-F767ZI $\left.{ }^{\circledR}\right)$.

The acquisition of DOS output and the ADC was handled in a single interrupt service routine, which was triggered by the data ready pin of the DOS. The timing information was generated by an internal $108 \mathrm{MHz}$ counter, which, in turn, was synchronised with an externally provided pulse-per-second (PPS) clock from a GPS receiver. The details of the implementation are available under open access conditions [11], [12]

The uncertainty of the absolute timestamp was evaluated by measuring a reference PPS signal stabilised to PTB's atomic clock with the DAU. Figure 2 shows the deviation of the timestamps from the expected value as determined by the DAU. That deviation is mainly caused by the short-term drift of the microcontroller's phase locked loop and the intrinsic jitter of the GPS's PPS signal. Overall, an accuracy better than 150 ns was achieved.

\subsection{Data analysis for sample time stamping}

Based on the described signal generation and data acquisition scheme, the system generates four times series of timestamped samples related to three different signal sources. The amplitudes and initial phases of these can be determined by appropriate sine

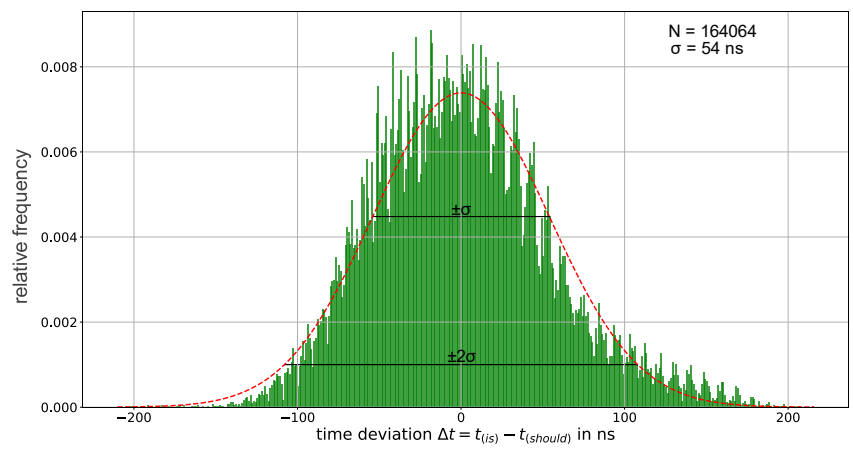

Figure 2. Distribution of the timestamp deviations determined using the DAU with reference to an atomic clock stabilised PPS signal over a monitoring period of 45 hours.

approximation methods (described below). Those time-series are:

i. The reference signal acquired by the ACS:

$y_{i, \text { ref }}\left(t_{i}\right)=\hat{y}_{\text {ref }} \cdot \sin \left(\omega t_{i, \mathrm{ACS}}-\varphi_{\text {ref }}\right)$.

ii. The sync signal acquired by the ACS:

$y_{i, \text { sync }}\left(t_{i, \mathrm{ACS}}\right)=\hat{y}_{\mathrm{sync}, \mathrm{ACS}} \cdot \sin \left(\omega t_{i, \mathrm{ACS}}-\varphi_{\text {sync,ACS }}\right)$.

iii. The sync signal acquired by the DAU:

$y_{k, \mathrm{sync}}\left(t_{k, \mathrm{DAU}}\right)=\hat{y}_{\mathrm{sync}, \mathrm{DAU}} \cdot \sin \left(\omega t_{k, \mathrm{DAU}}-\varphi_{\mathrm{sync}, \mathrm{DAU}}\right)$.

iv. The DOS signal acquired by the DAU:

$y_{l, \mathrm{DOS}}\left(t_{l, \mathrm{DOS}}\right)=\hat{y}_{\mathrm{DOS}} \cdot \sin \left(\omega t_{l, \mathrm{DOS}}-\varphi_{\mathrm{DOS}}\right)$.

Here, the index $i$ indicates that (i) and (ii) have the same time base and are synchronously sampled (by the ACS). In contrast (iii) and (iv) do need the same time base (from the DAU) but not necessarily synchronous sampling, hence the indices are $k$ and $l$.

The magnitude of the transfer function of the DOS is independent of the actual timing situation and can be directly calculated from (i) and (iv) as

$$
\hat{S}(\omega)=\frac{\hat{y}_{\mathrm{Dos}}}{\hat{y}_{\mathrm{ref}}} .
$$

Since (ii) and (iii) are records of the same source with only apparent but no real existing delay between $\varphi_{\text {sync,ACS }}$ and $\varphi_{\text {sync,DAU }}$, the time bases of ACS and DAU and thus the initial phases of the reference and the DOS can be linked.

The phase difference between $y_{\text {ref }}$ and $y_{\text {DOs }}$ is accordingly given by

$$
\Delta \varphi=\varphi_{\mathrm{DOS}}-\varphi_{\text {sync,Dau }}+\varphi_{\text {sync,ACS }}-\varphi_{\text {ref }} .
$$

It should be noted that the accuracy of that link is dependent on the timestamping accuracy of the DAU and not on the precision of the DOS's sample clock. As well as the uncertainty of the sync signal (iii) sampled by the DAU. The transfer function of the DAU ADC must be calibrated and taken into account [13]. On the contrary, the measurement scheme described above provides additional information on the precision of the DOS's sample clock, as described in Section 3.

\subsection{Series timestamping}

Some digital sensors/measuring systems do not provide a data ready signal for each sample but instead record a full time series of data after a given trigger signal. To calibrate such a kind of sensor (we will call it "time series sensor", TSS), precise knowledge of the relative time between the trigger and the excitation signal is needed. If the ACS does not provide such a 
synchronised trigger signal, separate analogue trigger logic, e.g. from a digital storage oscilloscope (DSO), can be used to derive and generate the trigger signal from an analogue sync signal like that mentioned in Section 2.1.

Figure 3 shows a schematic diagram of a setup to calibrate such a TSS. The ACS again acquires the generator signal $y_{\text {sync }}\left(t_{i}\right)$, see Section 2.1, as for an analogue DUT (orange arrow in Figure 3 into the ACS). The DSO (in our case a Rigol MSO5072) triggers, upon zero crossing of the same analogue sync signal (orange arrow in Figure 3 into the DSO) and generates a trigger signal which is connected to the TSS's trigger input (brown arrow in Figure 3). This starts the recording of the time series $y_{\mathrm{TSS}}\left(t_{\mathrm{TSS}}\right)$.

Note that due to noise, the DSO does not trigger perfectly at the zero crossing of the sync signal, and there is some additional processing delay within the DSO. However, the resulting actual trigger delay can be determined and corrected for, e.g. by sine approximation of the sync signal acquired simultaneously by the DSO and determining the actual trigger phase $\Delta \varphi_{\mathrm{DSO}, \text { TriggerNoise }}$.

To this end, in a dedicated setup, the DSO is used to sample its own trigger output simultaneously to the sine wave of the sync signal, which causes that trigger output. Based on the two simultaneously acquired traces, the delay between the zero crossing of the sync signal (e.g. determined by sine approximation) and the rising edge of the trigger can be determined. Due to inevitable noise, there will be a certain variation in the determined trigger delay.

In the actual setup, the trigger signal is emitted with a mean delay of $\tau_{\mathrm{DSO}}=236(1) \mathrm{ns}$. This was previously calibrated with a rectangle generator and the feedback of the trigger output to a second input channel of the DSO.

The use of a rectangle generator, providing a steep rising edge, was considered more precise than the sine approximation approach applied to the sync signal. However, it requires additional equipment.

\subsection{Data analysis for series timestamping}

Subsequent to the data acquisition described, a fourparameter sine approximation (FPSA) according to [6] is used to determine the angular frequency $\omega$, amplitude $\hat{A}_{T S S}$, initial phase $\varphi_{\text {TSS }}$ and offset $C_{T S S}$ of the TSS's output $y_{\mathrm{TSS}, \omega}\left(t_{\mathrm{TSS}}\right)$ (Note that due to the operation principle of a TSS, the time instances are unknown.) With these parameters, the magnitude response $\hat{S}(\omega)$ of its complex transfer function at the excitation frequency $\omega$ can be obtained directly from the amplitude of the reference signal $\hat{A}_{\text {ref }}(\omega)$ and the amplitude of the time-series signal $\hat{A}_{\mathrm{TSS}}(\omega)$

$$
\hat{S}(\omega)=\frac{\hat{A}_{\mathrm{TSS}}(\omega)}{\hat{A}_{\mathrm{ref}}(\omega)} .
$$

Note that the internal sampling frequency of the TSS, $f_{\mathrm{s} \text {,TSS }}$ may differ from its specified nominal sampling frequency $f_{\mathrm{s}, \mathrm{TSS} \text {,nominal }}$. Such differences lead to a deviation of the apparent excitation frequency determined by the FPSA as compared to the applied excitation frequency $\omega$.

A reduced internal sample rate $f_{\mathrm{s} \text {,TSS }}$ leads to an apparent increase of $\omega_{\text {TSS }}$, and accordingly an increased $f_{\mathrm{s} \text {,TSS }}$ leads to an apparent reduction of this frequency. The actual mean internal sample rate $f_{\mathrm{s}, \mathrm{TSS}}$ can then be determined as follows:

$$
f_{\mathrm{s}, \mathrm{TSS}}=f_{\mathrm{s}, \mathrm{TSS}, \text { nominal }} \cdot \frac{\omega}{\omega_{\mathrm{TSS}}},
$$

provided that the excitation frequency from the ACS, $\omega$, is well known.

The initial phase of the TSS signal $\varphi_{\text {TSS }}$ was determined using the four-parameter approximation and is thus linked to the actual period of the excitation signal. Therefore, no correction related to sample rate deviations of the measured initial phase is necessary.
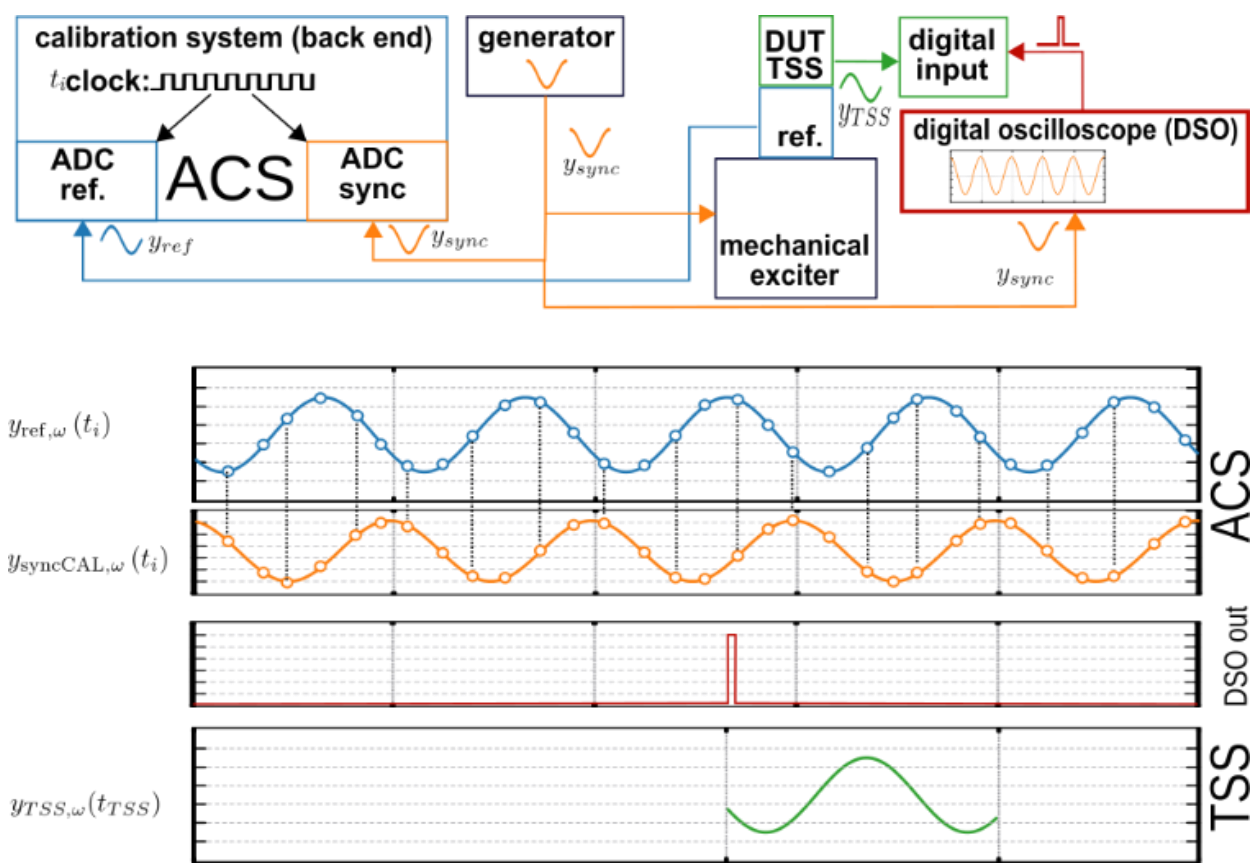

Figure 3. The ACS with a digital storage oscilloscope (DSO) as a trigger generator for the TSS. The DSO triggers on the zero crossing of the sync signal. The trigger output of the DSO, in turn, triggers the data acquisition of the TSS. The traces are the reference signal (blue), the analogue sinusoidal sync signal (orange), the trigger (brown) and the sampled time series of the TSS (green). 
Consequently, the phase response $\Delta \varphi$ of the complex transfer function of a TSS can be calculated as follows,

$$
\Delta \varphi=\varphi_{\mathrm{TSS}}-\Delta \varphi_{\mathrm{DSO}}-\Delta \varphi_{\mathrm{ACS}},
$$

using the phase difference $\Delta \varphi_{\mathrm{ACS}}$ between the reference and sync signal obtained by the analogue calibration system ${ }^{2}$.

Consequently, the complex transfer function $\hat{S}(\omega)$ is given by:

$$
S(\omega)=\hat{S}(\omega) \cdot \mathrm{e}^{-i \Delta \varphi}=\frac{\hat{A}_{\mathrm{TSS}}(\omega)}{\hat{A}_{\mathrm{ref}}(\omega)} \cdot e^{-i\left(\varphi_{\mathrm{TSS}}-\Delta \varphi_{\mathrm{DSO}}-\Delta \varphi_{\mathrm{ACS}}\right)} .
$$

\section{SAMPLE RATE PROBLEMS AND MITIGATION STRATEGIES}

Typical DOSs generate their internal sample rate with an internal clock unit based e.g. on an RC oscillator. Such simple clock generators are subject to drift depending on the operational temperature or supply voltage etc. Accordingly, the stability and precision of such clocks should be checked.

In [7] the effect of a biased sample frequency was described, and a rather elaborate correction method was provided. The calibration procedures presented in this article are independent of the internal sample clock by design, because they make use of external GPS-aligned timestamping. Even more, these procedures provide the direct means for a quantitative determination of sample clock accuracy and stability. This can be achieved at least in two ways:

\section{- Direct sample interval evaluation}

Based on the timestamps $t_{l, \mathrm{Dos}}$, the sample interval between each DOS sample pair can be calculated as $t_{l+1, \mathrm{DOS}}-t_{l, \mathrm{DO}}$ and compared to the nominal sample interval. This procedure and the resulting insights, of course, depend on the availability of independent precise and stable timestamps for each sample, which makes it unfeasible for the series timestamping.

\section{- Nominal frequency evaluation}

To get a more general estimate of the DOS sample rate precision and stability, it is sufficient to have a known, stable and precise single frequency excitation for the DOS. Accordingly, this method can be applied even without the complete calibration set-up. If the internal clock of the DOS deviates by a factor $\lambda$ from the laboratory clock, the DOS senses an external excitation with frequency $f_{\text {vib }}$ with an apparent frequency $f_{\mathrm{vib}, \mathrm{Dos}}$. The relation between the two is

$$
f_{\mathrm{vib}, \mathrm{DOS}}=\frac{f_{\mathrm{vib}}}{\lambda} \text {. }
$$

This is the equivalent situation to that described by equation (6) for series timestamping.

The apparent frequency sensed by the DOS can easily be determined by applying an FPSA [4] to the time series measured by the DOS. This algorithm does not only fit the amplitude and phase of a sinusoidal signal but iteratively reduces apparent frequency deviations starting with an initial frequency guess.

Note that in the case of sample timestamping the timestamps $t_{l}$ used for that fit have to be the nominal (deviating)

${ }^{2}$ It should be noted that any additional phase delays within the ACS known from conventional calibration have to be considered in the same fashion for the digitally enhanced system. timestamps calculated from the nominal sample rate of the DOS. Then, equation (9) yields the following for the real mean sample rate $f_{\text {Dos,real }}$ of the DOS

$$
f_{\text {DoS,real }}=\frac{f_{\text {DOS,nom }}}{\lambda} \text {. }
$$

\section{FIRST PRIMARY CALIBRATION RESULTS}

\subsection{The transfer function}

In order to test the above-mentioned concept, the DAU was used to perform a primary calibration of the z-axis of a 6-degreeof-freedom MEMS sensor of the type MPU-9250 following [6]. Figure 4 shows the situation in the ACS, i.e. the DOS mounted on the shaker's armature and the connected DAU. For the determined complex transfer function, the reference acceleration was measured by laser interferometry on top of the MEMS's housing. A second laser was targeted at the surface of the carrier breakout board right next to the DOS in order to investigate any relative motion due to the solder joints. This, however, is of no concern in this manuscript.

The generator signal driving the power amplifier for the shaker was used as the sync signal to link the ACS and the DAU. The calibration was performed in the frequency range from $10 \mathrm{~Hz}$ to $200 \mathrm{~Hz}$ with single frequency sine excitation and excitation levels between $2.5 \mathrm{~m} / \mathrm{s}^{2}$ and $100 \mathrm{~m} / \mathrm{s}^{2}$.

The MPU-9250 sensor is one of those that provide a "data ready" signal whenever the internal sampling process is finished. According to the previous description, the DAU acquired a sample of three acceleration values, three angular rate values and one temperature value for each trigger from the data ready signal and timestamped this set of values with an absolute time value. The sensor was running at a nominal sampling rate of $1000 \mathrm{~S} / \mathrm{s}$.

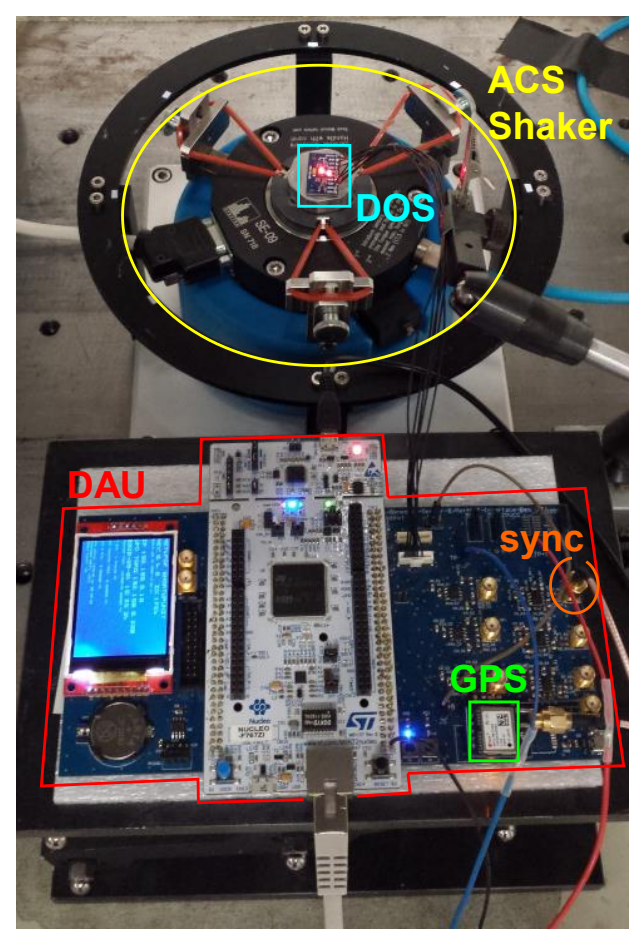

Figure 4. Primary calibration setup for z-axis of an MPU-9250 sensor. The bottom half shows the DAU with the STM32F7 development board in piggyback. In the top half, the MPU-9250-breakout board mounted on a steel adapter and screwed to the armature of an SE-09 shaker is shown. 

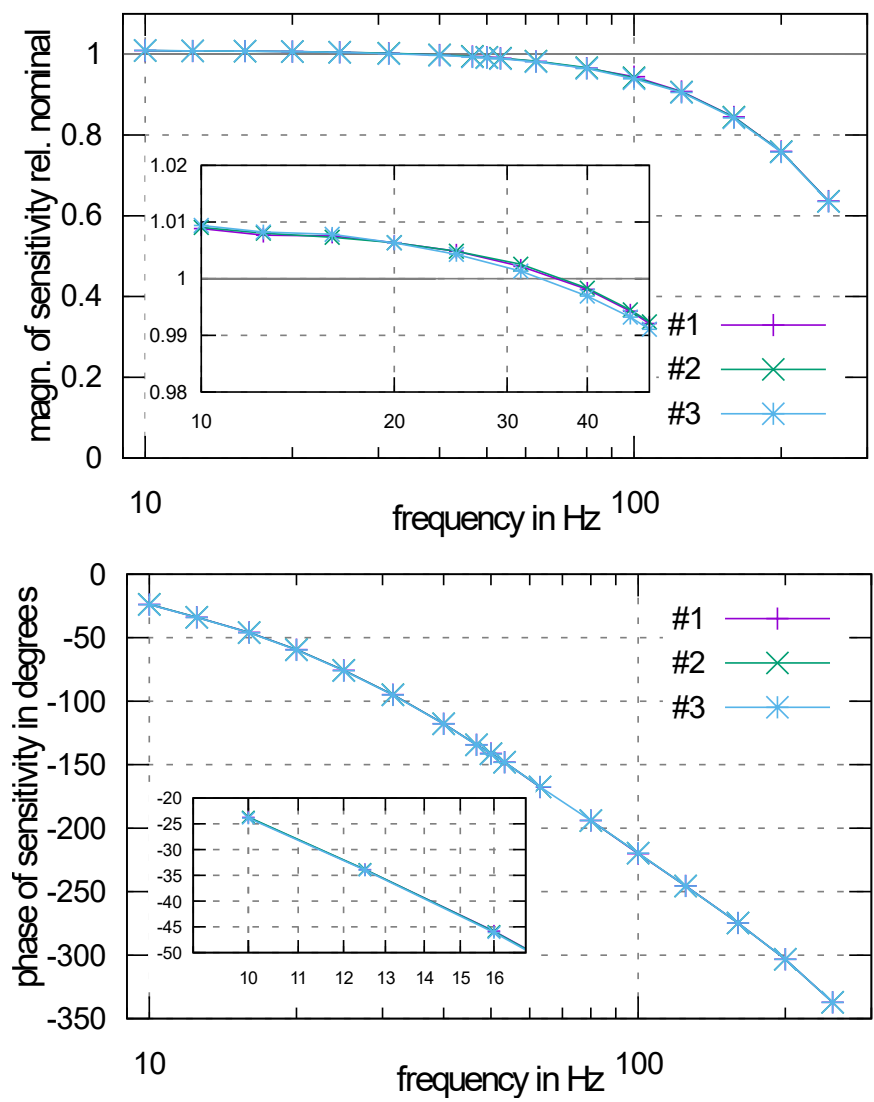

Figure 5. Magnitude (top) and phase (bottom) of the complex transfer function of an MPU-9250 sensor measured in 3 repetitions (\#1, \#2, \#3). The insert show details at low frequencies.

The analysis focused on the single (nominal) axis of excitation, which was the $\mathrm{z}$-axis.

By using the sync signal as described in Section 2.3 and sine fitting of the $\mathrm{z}$-axis values related to the absolute timestamps, it was possible to attain a traceable primary calibration of the magnitude and phase of the respective complex transfer function. The results are depicted in Figure 5 for three repeated measurements of the same unit.

A linear regression to the phase results for the lower frequencies suggests a group delay of the sensor of $8,1 \mathrm{~ms}$.

\subsection{The internal sampling}

In addition to this classic evaluation, the timestamping enables the evaluation of the stability of the sensor's internal sample clock and possible influencing quantities. In direct comparison to a $10 \mathrm{MHz}$ reference frequency distributed within PTB and traceable to the atomic fountain clocks of PTB, the utilised GPS-based timestamping revealed a mean sample interval of $996,96 \mu$ s for a measurement time of approx. $8000 \mathrm{~s}$. Over this time the sample interval length yielded a standard deviation of $21 \mathrm{~ns}$ and a spread of $335 \mathrm{~ns}$, where admittedly the quantization of the individual timestamps is $10 \mathrm{~ns}$ due to the counting frequency of $108 \mathrm{MHz}$ of the DAU. The distribution of the sample intervals is shown in the histogram in Figure 6.

From the visual appearance, it is evident that the distribution is not Gaussian and therefore not the usually supposed normal distribution. Rather than that, certain values of the sample interval are apparently strongly favoured over others. One cause for such an effect may be found in sensor-internal digital compensation processes in relation to temperature. As the MPU9250 also features an internal temperature probe, the

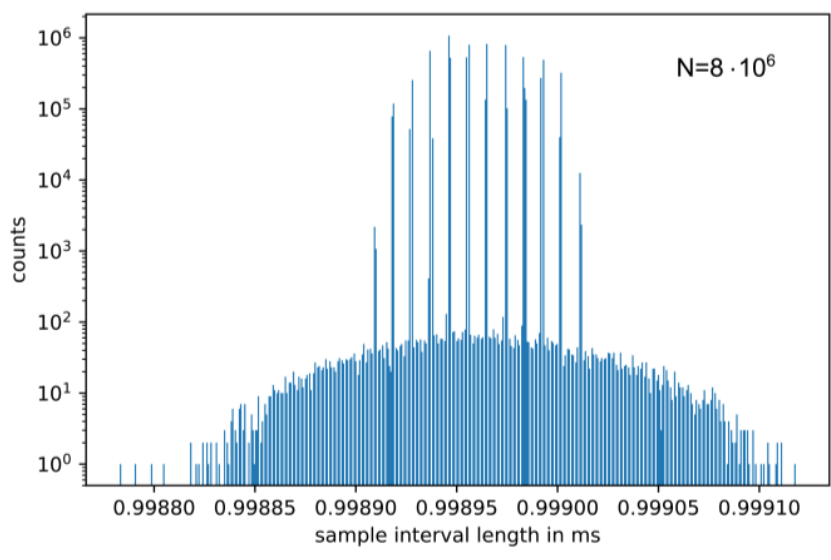

Figure 6. Distribution of sample intervals of an MPU-9250 DOS determined by sample timestamping. Counts are in logarithmic scale.

investigation of the correlation of the sample interval versus the temperature is an obvious next step.

Figure 7 depicts the relation of the sample interval over the apparent temperature as measured internally. It is evident that three to four of the preferred sample durations can be associated with a certain temperature and that, in general, the average sampling frequency falls (slightly) with rising temperature. For an in-depth understanding of this peculiar relation, a detailed knowledge of the internal processes of the sensor is probably necessary.

Nevertheless, it remains to be noted that the application of sample timestamping with the newly presented DAU could demonstrate that the statistical properties of the sampling unit of this sensor are far from the typically expected uniform or normal distribution.

In order to compare this finding with some other DOS, a Bosch BMA280 was calibrated in an identical setting, and the measured sample intervals were subject to the same processing. Figure 8 depicts the results.

In this case the distribution is split into three parts with a prioritised centre part which has three orders of magnitude more counts than the side lobes. Such a shape makes the usual characterisation with a standard deviation almost meaningless. Why such a peculiar distribution is generated becomes clearer on closer inspection of the time series of sample intervals given in Figure 9.

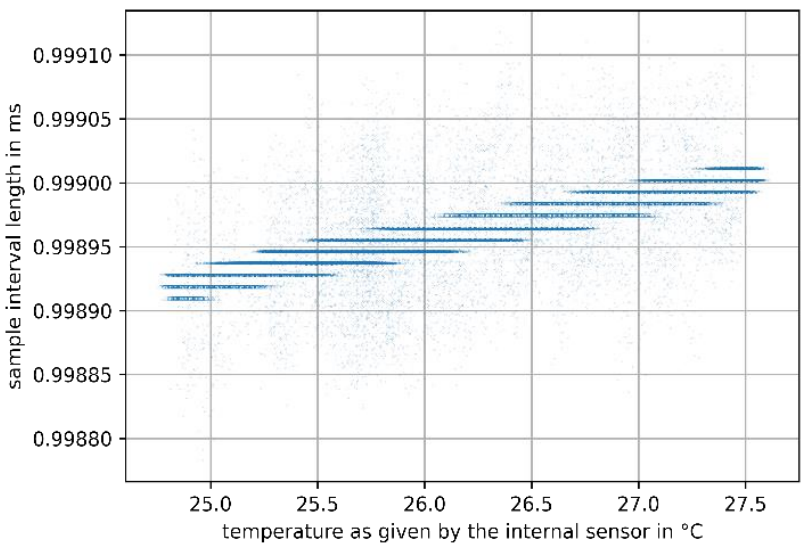

Figure 7. Relation of sample interval and sensed temperature of the MPU9250 sensor. 


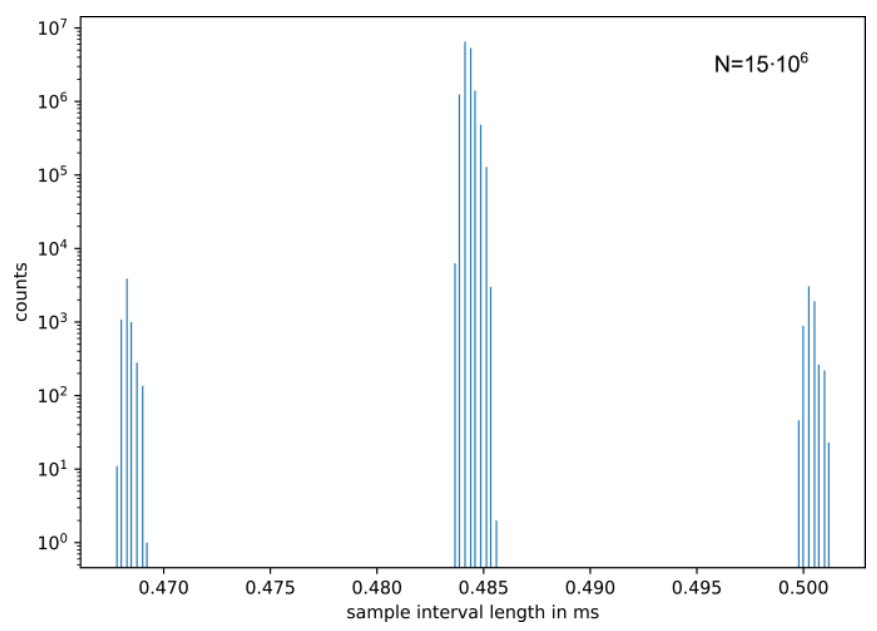

Figure 8. Distribution of sample interval lengths of a BMA280 DOS determined by sample timestamping. Counts are in logarithmic scale.

As seen in the upper chart, the vast majorities of the sample intervals are close to the mean value of $485 \mu$ s. However, periodically, i.e. approximately every $1.1 \mathrm{~s}$ or 2200 samples, there is a double spike. The close-up of one of those features in the lower chart of the figure discloses a combination of a delayed followed by a premature sample. Presumably, some internal process of the digital part of the sensor, like an adaptive digital filter or an adjustable compensation, takes some excessive computation time, slowing down either the sampling process or the delivery (data ready) of the analogue quantity. In order to keep the mean sample rate constant, the delay is followed by an abridged sample interval compensating the delay.

\section{MEASUREMENT UNCERTAINTY}

This article is not dedicated to an in-depth discussion and evaluation of the measurement uncertainty budget associated with the new hardware and methodology. This will need more research in the various components involved and probably is worth an article on its own. Nevertheless, we want to briefly address a few issues that were found during the research work so far.

\subsection{Magnitude of the transfer function}

The magnitude calibration is a rather straight forward process for the DOS and in terms of measurement uncertainty very similar to conventional work with AD-converted output voltages. A significant difference is, however, that opposed to conventional set-ups, no gain adjustment by using external

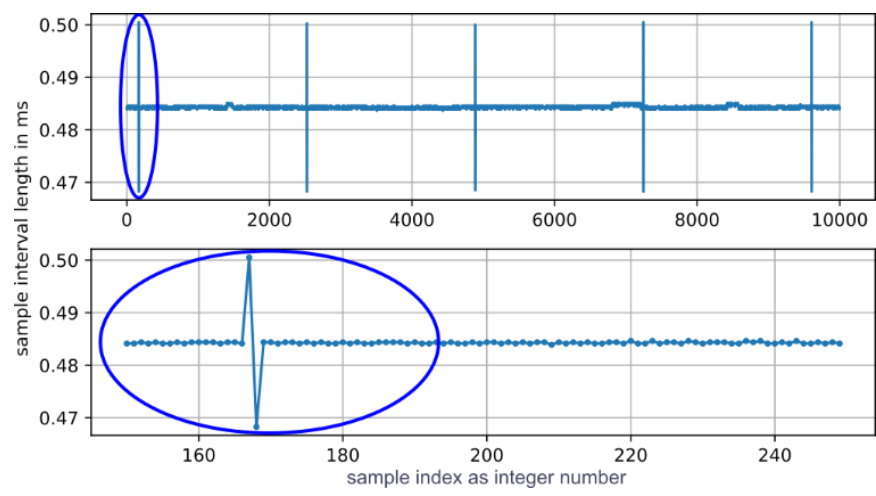

Figure 9. Sample interval length over time plot for the BMA280 sensor. The Lower plot is a zoom-in on the upper overview. amplifiers is possible. This results in a fixed signal resolution that is linked to the embedded ADC and has to be considered in the measurement uncertainty of the DOS especially for excitations in the lower amplitude range.

\subsection{Phase of the transfer function}

The phase of the transfer function is the actual challenge in calibration and therefore the focus of this article. Internally the measurement uncertainty of the phase is relying on the quality of the embedded oscillator and some firmware aspects of the DOS. This was demonstrated by the measurement-analysis of the previous section 4 . While the influence of a low-quality oscillator may be mitigated by precise timestamping during calibration, such an approach is usually not feasible for the typical application of DOS. Here, the nominal sampling rate is assumed.

In addition to these components of measurement uncertainty of phase intrinsic to the DOS, the hardware of the DAU and the methodology add their own components.

Of course, the phase or group delay associated with the sampling of the sync-signal by the DAU has to be calibrated, as this is no longer controlled by the ACS. The best estimate of this group delay can be corrected, but an uncertainty remains from this calibration. This was briefly addressed in sections 2.2 and 2.4 for the work presented here.

Additional phase measurement uncertainty is inflicted by the noise on the sync signal which links the time-bases of ACS and DAU. This is strongly dependent on the source available as sync signal and in particular its signal-to-noise ratio. This noise degrades the phase accuracy on both sides, the ACS and the DAU. However, in the described set-up it can hardly be avoided.

\section{CONCLUSION AND OUTLOOK}

Conventional analogue accelerometer calibration systems are not capable of calibrating modern digital output sensors. However, an extension providing the necessary digital interface and analogue input for a suitable reference signal can extend the capabilities to the magnitude and phase calibration of the complex frequency response of such sensors. The typically independent internal sampling of the DOS poses a special challenge in the calibration as well as a new source of measurement uncertainty and a new specification that has to become part of the calibration routine and the calibration result.

With two different approaches, it is possible to calibrate devices that deliver separate samples as well as devices that buffer a series of samples after a single trigger and deliver the whole series at once. Provided that the calibration laboratory is equipped with a precise and stable timestamp source, like a GPSaligned clock, simple procedures lead to detailed insights concerning the internal timing of the DOS and their dependencies on environmental conditions.

It turns out that simple classical assumptions about characteristics like the accuracy and jitter of the internal sampling units can be misleading. However, the application of external timestamping, as demonstrated, gives a clear and traceable quantification of these properties.

Within the European project titled Met4FoF, a cross validation in terms of an interlaboratory comparison between different participants is planned. In order to address the question of consistency of the results, a further evaluation of additional measurement uncertainty components involved with the use of the DOS will be included. An existing calibration system will be equipped with a GPS based absolute time base so that the phase of the transfer function can be determined without a reference 
signal, but by means of the absolute time. By comparison with this system, the solution presented here will be evaluated with respect to uncertainty.

The intrinsic timing properties, in particular, add a new dimension to measurement uncertainty estimation. In addition, most MEMS accelerometers on the market are multi-component devices. The question of dynamic crosstalk may therefore add another complication, where the static (DC) crosstalk has already been studied in other publications such as [7], [8].

\section{ACKNOWLEDGEMENT}

This project 17IND12 Met4FoF has received funding from the EMPIR programme co-financed by the Participating States and from the European Union's Horizon 2020 research and innovation programme

\section{REFERENCES}

[1] N. Helwig, E. Pignanelli, A. Schütze, Condition Monitoring of a Complex Hydraulic System Using Multivariate Statistics, Proc. I2MTC-2015 - 2015 IEEE International Instrumentation and Measurement Technology Conference, paper PPS1-39, Pisa, Italy, May 11-14 (2015), https://ieeexplore.ieee.org/document/7151267

[2] B. Seeger, Th. Bruns S. Eichstädt, Methods for dynamic calibration and augmentation of digital acceleration MEMS sensors. 19th International Congress of Metrology, 22003, Paris, France (2019), https://cfmetrologie.edpsciences.org/articles/metrology/pdf/20 19/01/metrology cim2019 22003.pdf

[3] S. Eichstädt, M. Gruber, A.P. Vedurmudi, B. Seeger, Th. Bruns, G. Kok, Toward Smart Traceability for Digital Sensors and the Industrial Internet of Things. Sensors 21(6) (2021), DOI: $\underline{10.3390 / \mathrm{s} 21062019}$

[4] Guideline DKD-R 3-2: Calibration of Conditioning Amplifiers for Dynamic Application, Physikalisch-Technische Bundesanstalt, DKD, Braunschweig, Gemany (2019), DOI: $\underline{10.7795 / 550.20190425 \mathrm{EN}}$
[5] G. D'Emilia, A. Gaspari, F. Mazzoleni, E. Natale, A. Schiavi, Calibration of tri-axial MEMS accelerometers in the lowfrequency range - Part 1: comparison among methods J. Sens. Sens. Syst., 7, 245-257, 2018, DOI: $10.5194 /$ isss-7-245-2018

[6] IEEE 1057-2017 - IEEE Standard for Digitizing Waveform Recorders, Standard, Institute of Electrical and Electronics Engineers, New York, NY, USA (2018). Online [Accessed 8 September 2021] https://standards.ieee.org/standard/1057-2017.html

[7] W.-S. Cheung, Effects of the sample clock of digital-output MEMS accelerometers on vibration amplitude and phase measurements, Metrologia, 57 (1) (2020), DOI: $10.1088 / 1681-7575 /$ ab5505

[8] ISO 16063-11:1999, Methods for the calibration of vibration and shock transducers - Part 11: Primary vibration calibration by laser interferometry, International Organization for Standardization, Geneva, CH (1999).

[9] A. Prato, F. Mazzoleni, A. Schiavi, Traceability of digital 3-axis MEMS accelerometer: simultaneous determination of main and transverse sensitivities in the frequency domain, Metrologia, 57 (3) (2020) p. 035013. DOI: $10.1088 / 1681-7575 /$ ab79be

[10] M. Y. A. Michael Gaitan, J. Geist, Analysis and Protocol for Characterizing Intrinsic Properties of Three-Axis MEMS Accelerometers using a Gimbal rotated in the Gravitational Field, Proc. of the IMEKO 23rd TC3, 13th TC5 and 4th TC22 International Conference, Helsinki, Finland, 30 May - 1 June 2017, 3 pp. Online [Accessed 8 September 2021] https://www.imeko.org/publications/tc22-2017/IMEKOTC22-2017-015.pdf

[11] Software for the Met4FoF Smartup Unit V2. Online [Accessed 8 September 2021] https://github.com/Met4FoF/Met4FoF-SmartUpUnit

[12] PCB Layout of the Met4FoF Interface Board. Online [Accessed 8 September 2021] https://circuitmaker.com/Projects/Details/Benedikt-Seeger2/Met4FoF-Interface-Board

[13] B. Seeger, L. Klaus D. Nordmann, Dynamic calibration of digital angular rate sensors, ACTA IMEKO 9 (2020) 5, pp. 394-400. DOI: $\underline{10.21014 / \text { acta imeko.v9i5.1008 }}$ 